\title{
Functional analysis of the Drosophila twist promoter reveals a dorsal-binding ventral activator region
}

\author{
Duojia Pan, Jian-Dong Huang, and Albert J. Courey ${ }^{1}$ \\ Department of Chemistry and Biochemistry, University of California, Los Angeles, California 90024-1569 USA
}

\begin{abstract}
twist is one of the earliest expressed zygotically active genes required for dorsal-ventral pattern formation in the Drosophila embryo. Genetic studies suggest that this gene is activated in the ventral part of the blastoderm by maternally expressed dorsal gene product. Using P-element-mediated germ-line transformation, we have mapped a small $(260 \mathrm{bp})$ dorsal-dependent ventral activator region (VAR) in the 5 '-flanking region of the twist promoter that can direct the early ventral expression of a heterologous promoter. The VAR contains binding sites for a number of proteins present in extracts of Drosophila embryos. One of these sites bears homology to known binding sites for the dorsal transcription factor and is specifically bound by bacterially expressed dorsal protein. Furthermore, a 37 -bp deletion that removes the dorsal-binding sequences abolishes the ventral-specific activity of the twist promoter constructs. Our data also show that additional sequences within the VAR are required to render the dorsal-binding sites functional. Finally, reverse genetic and biochemical data suggest that the transcription factor, encoded by the zeste gene may help control the overall level, if not the pattern, of twist expression.
\end{abstract}

[Key Words: twist; dorsal; transcription; Drosophila embryogenesis; dorsal-ventral pattern formation]

Received June 26, 1991; revised version accepted August 12, 1991.

A large number of genes that influence the process of Drosophila embryogenesis have been identified, and many of them have been found to encode DNA-binding proteins that serve to control the transcription of downstream genes (Ingham 1988; Govind and Steward 1991). Intensive genetic and molecular analysis has succeeded in organizing these genes into intricate regulatory hierarchies. Most developmentally important genes can be grouped into one of two classes: (1) those that determine cell fate as a function of position along the anteriorposterior axis and (2) those that determine cell fate as a function of position along the dorsal-ventral axis of the early embryo. The former class specifies the segmental structure of the embryo, whereas the latter set determines the germ layers of the developing organism. The initial designation of position along the dorsal-ventral axis involves the action of a large set of maternally active genes, most of which belong to the "dorsal group" of maternal effect genes. The role of these genes is to generate an activity gradient of the product of the dorsal (dl) gene (itself a member of the dorsal group) emanating from the ventral midline of the blastoderm embryo. It is this activity gradient of the $d l$ protein that specifies the dorsal-ventral coordinate axis of the embryo.

The $d l$ gene exhibits extensive sequence similarity to

${ }^{1}$ Corresponding author. the nuclear proto-oncogene c-rel (Steward 1987) and the gene encoding the human transcription factor NF- $\mathrm{KB}$ (Ghosh et al. 1990, Kieran et al. 1990), and thus is believed to encode a transcription factor. In support of this idea, it has been shown that the $d l$ protein is a DNAbinding protein that specifically recognizes sequences similar to those bound by NF-kB (Ip et al. 1991). Furthermore, in accord with genetic data suggesting that $d l$ is a repressor of zerknüllt (zen), the zen ventral repressor element was found to contain binding sites for the $d l$ protein (Ip et al. 1991). The site of action of the $d$ l protein is the nucleus (Steward 1987), and a number of researchers have demonstrated that the activity gradient of the $d l$ protein is caused by a gradient of nuclear localization in the syncytial blastoderm embryo (Roth et al. 1989; Rushlow et al. 1989; Steward 1989). Specifically, the dl protein is transported efficiently into ventral nuclei, less efficiently into lateral nuclei, and not at all into dorsal nuclei.

Partially in response to the $d l$ activity gradient, a number of early zygotically active genes are expressed in discrete domains along the dorsal-ventral axis of the embryo (Govind and Steward 1991). These include the dorsally active genes zen and decapentaplegic $(d p p)$, which are initially expressed in the anlagen of the dorsal ectoderm and the amnioserosa, and the ventrally active genes snail (sna) and twist (twi), which are initially expressed in the mesodermal anlagen. Genetic evidence 
strongly suggests that $d l$ is a repressor of zen and $d p p$ and an activator of $t w i$ and sna. Thus, studies of $d l$ should reveal information about the mechanisms of both transcriptional activation and repression.

As indicated above, twi is first activated in the ventral $20-25 \%$ of the nuclei of the blastoderm embryo in the mesodermal anlagen (Thisse et al. 1987, 1988). This gene is also expressed in the anterior and posterior poles of the early embryo. Genetic analysis demonstrates that the early ventral activation of $t w i$ is completely dependent on $d l$ (Thisse et al. 1987). twi expression is absolutely required for gastrulation and for the differentiation of mesoderm. twi appears to encode a helix-loop-helix transcription factor (Murre et al. 1989); therefore, it presumably carries out its functions in gastrulation and cell differentiation by activating or repressing downstream genes at the transcriptional level.

To help elucidate the mechanisms that generate the spatial and temporal pattern of twi transcription, we have used P-element-mediated germ-line transformation to map cis-regulatory transcriptional control elements in this gene. In addition, we have initiated a biochemical analysis of the protein factors that interact with twi control regions. These experiments have allowed us to identify a number of elements in the $5^{\prime}$-flanking region of the gene that contribute to the normal twi pattern of expression. In particular, we have mapped a small region that directs the ventral activation of $t w i$. This region is bound by several nuclear proteins in the Drosophila embryo, including the $d l$ protein. Our results suggest that $d l$ bound to this region interacts either directly or indirectly with other DNA-bound regulatory factors to activate $t w i$ in the presumptive mesoderm.

\section{Results}

5 -Deletion analysis of the twi promoter defines a small region sufficient for ventral-specific expression

In Figure $1(A, B)$, we show whole-mount in situ hybridizations in which wild-type blastoderm stage Drosophila embryos have been probed with a digoxigenin-labeled probe containing the $t w i$-coding region to reveal the spatial distribution of the twi mRNA. In agreement with data published previously (Thisse et al. 1987, 1988), we find that $t w i$ is first expressed in the ventral $20-25 \%$ of the blastoderm (in the mesodermal anlagen) and that this early domain of expression also includes the anterior and posterior poles of the embryo.

To locate regions of the twi gene sufficient to direct this ventral-specific RNA synthesis, we created a series of transgenic fly lines bearing various portions of the $t w i$ 5 '-flanking region fused to the lacZ-coding region. In our first series of experiments, we examined a set of 5 '-deletion mutants extending from a variable position upstream of the $t w i$ transcriptional start site to a fixed position just downstream of the $t w i$ initiation codon $(+236)$. Embryos from the various transgenic lines were fixed and subjected to in situ hybridization with a digoxigenin-labeled probe specific for the lacZ mRNA. For each construct, the embryos shown are representative of multiple independently derived transgenic fly lines. A construct containing $5 \mathrm{~kb}$ of 5 '-flanking sequences fused to the lacZ-coding region gives strong and specific ventral expression in the cellular blastoderm that is essentially indistinguishable from endogenous twi expression $(-5000$ twi/lacZ; Fig. 1C,D). Likewise, deletion mutants containing only $1829 \mathrm{bp}(-1829$ twi/lacZ; Fig. $1 \mathrm{E}, \mathrm{F}), 920$ bp $(-920$ twi/lacZ; Fig 1G,H), or 648 bp $(-648$ twi/lacZ; Fig.1I,J) of 5 '-flanking sequences exhibit specific ventral expression (although, as discussed below, the exact expression patterns of $-920 \mathrm{twi} / \mathrm{lacZ}$ and $-648 \mathrm{twi} / \mathrm{lacZ}$ differ somewhat from those of the longer constructs). In contrast, a construct containing only 269 bp of 5'-flanking sequences does not exhibit ventral expression in the cellular blastoderm $(-269$ twi/lacZ; Fig. $1 \mathrm{~K})$. Thus, these experiments (summarized in Fig. 1L) suggest that the region extending from -648 to -269 relative to the transcriptional start site contains an element that is required for ventral activation of twi in the Drosopohila embryo.

A detailed examination of the embryos bearing the $-920 \mathrm{twi} / \mathrm{lacZ}$ or $-648 \mathrm{twi} / \mathrm{lacZ}$ constructs (Fig. 1G-J) shows that they exhibit expression patterns that differ in a number of ways from those observed with the longer constructs (Fig. 1C-F). First, the level of activity is somewhat reduced. Second, the domain of expression does not include the anterior or posterior poles of the embryo (see arrows, Fig. 1E,G). Third, the width of the ventral lacZexpressing domain is narrowed (see arrows, Fig. 1F,H). Finally, these promoter constructs exhibit aberrant expression in the head region, which appears slightly later than the ventral expression (late stage 5; see arrow, Fig. 2A). Thus, the region upstream of $-920 \mathrm{bp}$ seems to contain regulatory elements required to direct expression in the termini, to strengthen and broaden the ventral domain and to repress aberrant expression in the head region.

To determine whether the observed ventral expression is dependent on $d l$, homozygous males containing twi/ lacZ fusion constructs were crossed to females homozygous for a null mutation in $\mathrm{dl}$. The resulting embryos were analyzed as described above, and one example (with the -920 twi/lacZ construct) is shown in Figure 2. Similar results were observed with other constructs (data not shown). In the absence of $d l$ gene product (Fig. 2B), the embryos lack the ventral domain of $l a c Z$ expression observed in the wild-type background (Fig. 2A). Thus, the 5 '-deletion series appears to define a small $\mathrm{dl}$-dependent ventral activator element. Interestingly, unlike the ventral-specific activity, the aberrant head-specific expression characteristic of some of the deletion constructs seems to be independent of $d l$ and is retained in the $\mathrm{dl}^{-}$ background (see arrows, Fig. 2B,C). The $d l$-independent head expression was observed with multiple transformants of the -920 twi/lacZ and -648 twi/lacZ constructs but not with the longer constructs /which use the same vector). This suggests that this expression is an inherent property of certain twi promoter deletion $\mathrm{mu}$ tants and not a position or vector effect. 


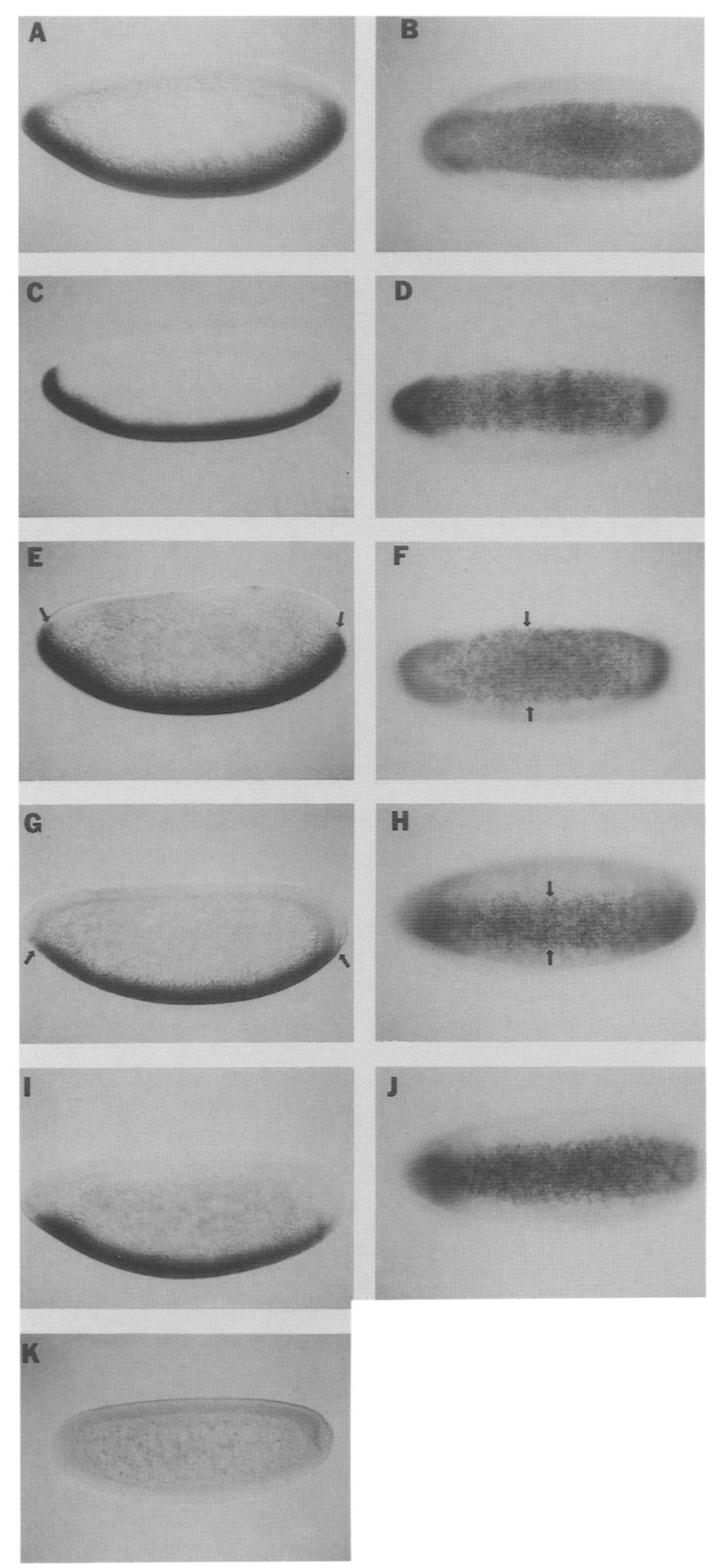

The ventral activator can direct the expression of a heterologous promoter

To determine whether the ventral activator can work in conjunction with a heterologous promoter, we fused various portions of the 660 -bp proximal promoter region to a basal level hsp 70 promoter (Fig. 3). Embryos from transgenic fly lines bearing these constructs were collected, and the expression patterns of the lacZ transgenes were assessed as described above. Sequences ex-

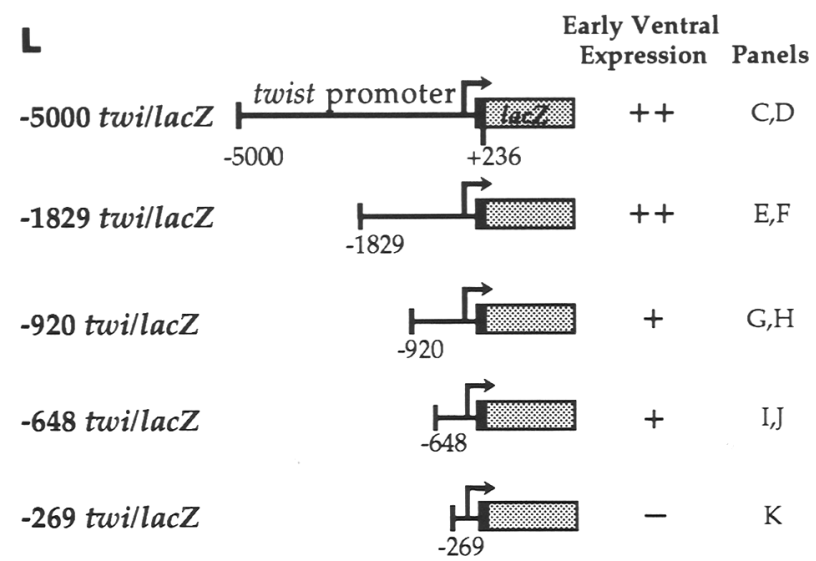

Figure 1. Expression of $t w i / l a c Z$ transgenes in Drosophila embryos. Patterns of expression were revealed by in situ hybridization for twi $(A$ and $B)$ or $\operatorname{lac} Z(C-K)$ mRNA. Stage 5 (cellular blastoderm) embryos are shown in both sagittal $(A, C, E, G, I, K)$ and ventral $(B, D, F, H, I)$ views. The various constructs used are shown in $L$. All frames show anterior to the left; in the sagittal views, dorsal is up. $(A, B)$ Wild-type (Oregon $\mathrm{R}$ ) embryos probed to reveal normal $t w i$ pattern of expression. $(C, D)-5000$ twi/ lacZ. $(E, F)-1829$ twi/lacZ. $(G, H)-920$ twi/lacZ. $(I, /)-648$ twi/lacZ. In contrast to the $-5000 \mathrm{twi} / \mathrm{lacZ}$ and $-1829 \mathrm{twi} /$ lac $Z$ constructs, the domains of lacZ expression in the -920 $t w i / l a c Z$ and the $-648 \mathrm{twi} / \mathrm{lac} Z$ constructs do not include the termini (arrows in $E, G$ ). Also, these latter two constructs exhibit ventral domains of $l a c Z$ expression that are narrower /cf. arrows in $F, H$ ) and of lower intensity. $(K)-269$ twi/lacZ (no staining).

tending from -660 to $-55(t w i / h s p-1)$ were found to direct ventral-specific expression of the $h s p 70$ basal level promoter element (Fig. 3A), demonstrating that the ventral activator can mediate the ventral-specific expression of a heterologous promoter. This expression is approximately five- to eightfold weaker than that observed for constructs containing the normal twi promoter and 648 or $920 \mathrm{bp}$ of 5 -flanking sequences. Thus, the constructs shown in Figure 3 were stained longer than those in Figure 1.

When the 605-bp element extending from -660 to $-55(t w i / h s p-1)$ was deleted from the $3^{\prime}$ end, it was found that deletion to -205 (twi/hsp-2; Fig. 3D,E) or -269 (twi/hsp-3; Fig. 3F,G) resulted in a sharp drop in overall promoter strength, but weak ventral-specific activity was retained. Further deletion to -306 (twi/hsp-4) resulted in the complete loss of early ventral activity. Thus, these 3 ' deletions define a 37-bp region (from -306 to -269 ) that contains sequences essential for ventral activation. When the 605 -bp element was deleted from the $5^{\prime}$ end, a deletion to -537 (twi/hsp-7) was found to have no effect (Fig. 3B,C), whereas a deletion to $-323(t w i / h s p-8)$ eliminated ventral expression. Taken together, these results (which are summarized in Fig. $3 \mathrm{H}$ ) suggest that the 605-bp region contains at least two functional units (Fig. 4B). Part or all of the first unit (which we have named element $z$ ) lies between -55 and -205 . This element is not required for ventral-specific activity 

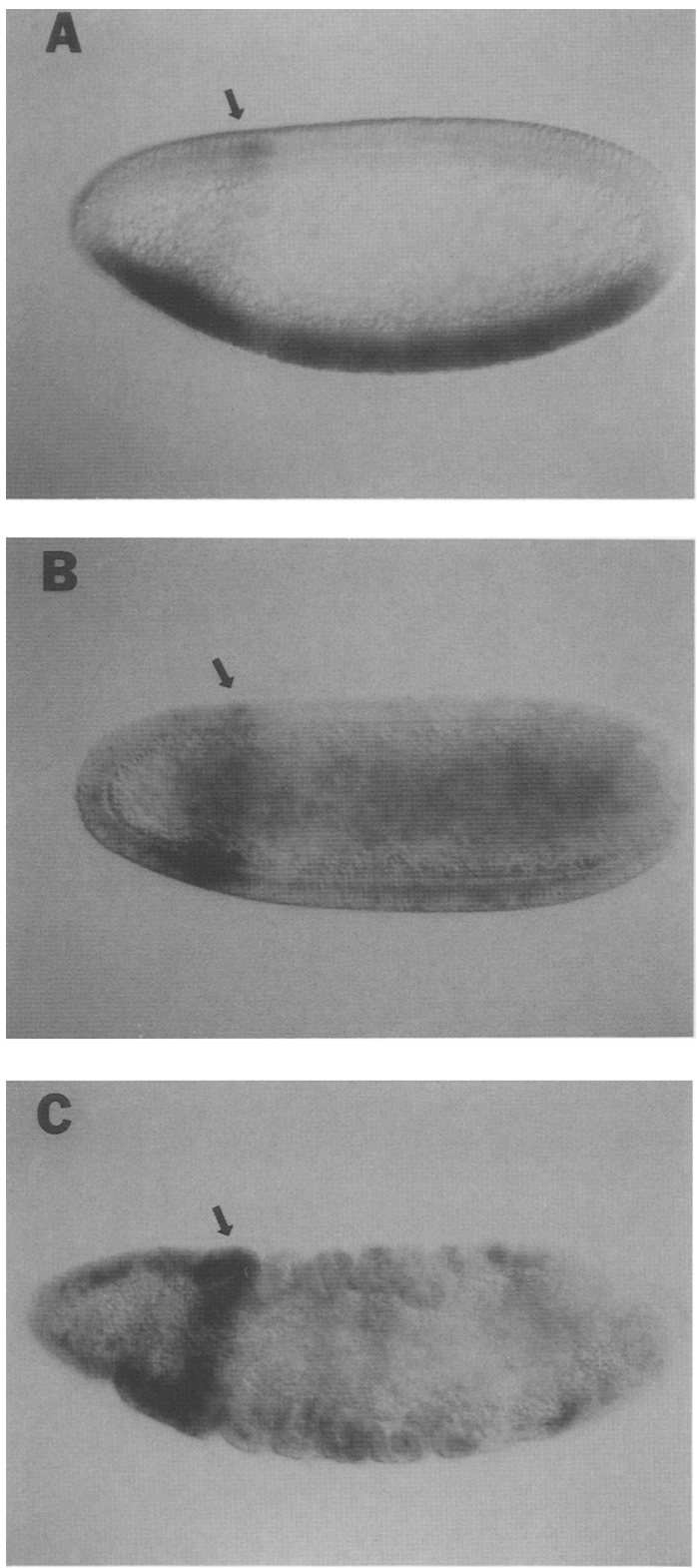

Figure 2. $d 1$ dependence of the VAR. Expression pattern of the $-920 t w i / l a c Z$ construct was examined in wild-type $(A)$ and $d l^{-}$backgrounds $(B, C)$ by in situ hybridization to lac $Z$ mRNA. Embryos are oriented so that anterior is to the left and dorsal is up. The ectopic expression in the head region (arrows) comes on later (late stage 5) than the ventral expression (easily seen at early stage 5 ). This ectopic head region expression was observed in $-920 \mathrm{twi} / \mathrm{lacZ}$ and $-648 \mathrm{twi} / \mathrm{lacZ}$ constructs, but not in the longer constructs. Note that the ventral expression $(A)$ is absent in the $\mathrm{dl}^{-}$background $(B)$. However, the head expression remains and is retained later in embryogenesis $(C)$.

but influences overall promoter strength. The second functional element lies somewhere between -537 and -269 and constitutes a ventral activator region (VAR), as it is able to mediate the ventral activation of the heterologous $h s p 70$ basal level promoter.
To initiate an analysis of the trans-acting factors responsible for the ventral-specific expression of $t w i$, we scanned the $600-b p$ proximal promoter region using a footprinting assay. Probes covering the entire region were incubated with crude Drosophila embryo extracts before being subjected to DNase I digestion. Representative footprinting data is shown in Figure 4A and summarized in Figure 4,B and C. The footprint analysis reveals three prominent clusters of footprints (Fig. 4B). One cluster consists of four strong protections between -10 and -120 , three of which lie within the boundaries of element $z$. Three protections are found between -240 and -312 , a region that overlaps the $3^{\prime}$ boundary of the VAR. Finally, the third cluster includes three regions of protection that are located between -604 and -484 , a region that overlaps the $5^{\prime}$ boundary of the VAR.

The activity of element $z$ may be mediated by the zeste transcription factor

As mentioned above, the proximal $120 \mathrm{bp}$ of the twi promoter was found to contain four strongly protected regions of $\sim 10-15$ bp each, three of which lie within the bounds of element $z$. Inspection of the protected sequences (Fig. $4 \mathrm{C}$ ) reveals that they each contain either a five of six or a six of six match for the binding site of the transcription factor encoded by the zeste gene (Biggin et al. 1988). These sites are protected by a homogeneous preparation of Drosophila zeste protein purified on the basis of its affinity for sites found in the Ultrabithorax $(U b x)$ promoter (data not shown). Thus, the activity of element $z$ may be mediated, at least in part, by the zeste transcription factor.

Distant regulatory elements are not normally active in conventional in vitro transcription systems. However, as the zeste-binding sites lie within the 120 -bp region proximal to the transcriptional start site, we were encouraged to examine the activity of these sites in an in vitro transcription system. Thus, we analyzed a series of 5 '-deletion mutants for transcriptional activity in an extract derived from Drosophila embryos (Fig. 5). Deletion of the most distal zeste site has little or no effect on promoter activity (Fig. 5A, cf. lanes 1 and 2 with lanes 3 and 4). In contrast, a deletion that leaves only the two proximal zeste sites intact (lanes 5,6$)$ causes a sharp reduction in promoter activity. The further deletion of one of the two remaining zeste sites does not cause a further reduction in promoter activity (lanes 7,8$)$. Thus, the region containing three of four zeste-binding sites seems to be required for efficient in vitro transcription.

The experiments described in the preceding paragraph were carried out with transcription extracts prepared by the method of Heiermann and Pongs (1985; HP extracts). A different result is obtained if we use extracts prepared by the method of Soeller et al. (1988; SK extracts). In this case, high levels of transcription are observed for all the deletion templates and there does not seem to be any dependence on sequences upstream of -51 (Fig. 5B, cf. lanes 1 and 2). It has recently been demonstrated that a major difference between these two types of extracts is 


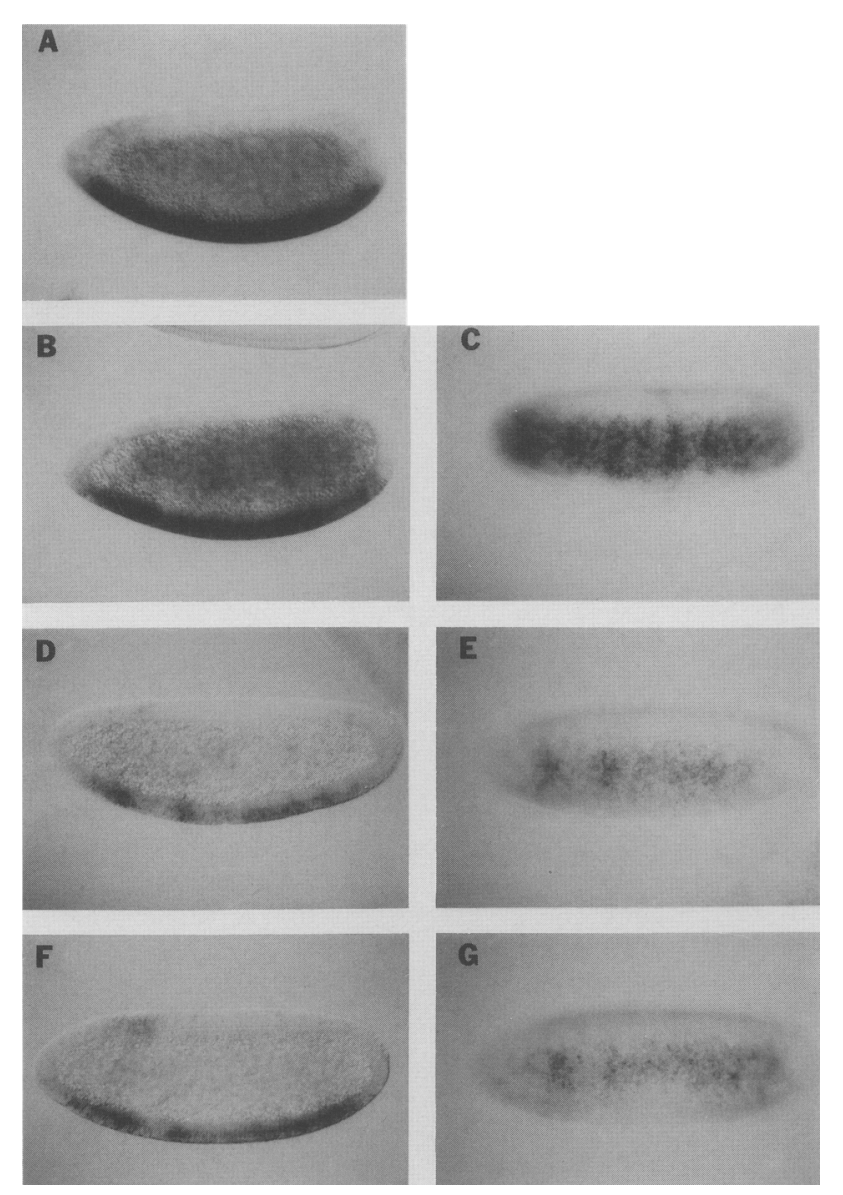

the presence of histone $\mathrm{Hl}$ in the HP extracts and the absence of this protein in SK extracts (Croston et al. 1991; Kerrigan et al. 1991). We have found that the addition of $\mathrm{H} 1$ to the SK extracts has two effects on the in vitro activity of the twi promoter (lanes 3-7). First, this protein has an overall negative effect on promoter activity. (This is also true of the Adh proximal promoter, which was used as an internal control in these in vitro transcription experiments.) Second, the addition of this protein restores the activity of sequences upstream of -51 . Thus, in the HP extracts, the zeste-binding sites seem to act to overcome a generalized repression by histone $\mathrm{H} 1$. This conclusion is strengthened by the finding that in the presence of excess DNA (lanes 10-12), the HP extracts behave similarly to the SK extracts (i.e., high levels of expression are observed both in the absence and presence of multiple zeste-binding sites). This is presumably because free $\mathrm{Hl}$, which is a nonspecific DNA-binding protein, is titrated out of solution by excess DNA.

The essential 37-bp segment of the VAR contains binding sites for the $\mathrm{dl}$ transcription factor

As noted above, the VAR is sufficient to drive the $d l$ dependent ventral expression of a heterologous promoter. Ip et al. (1991) have shown that $d 1$ is a sequence-

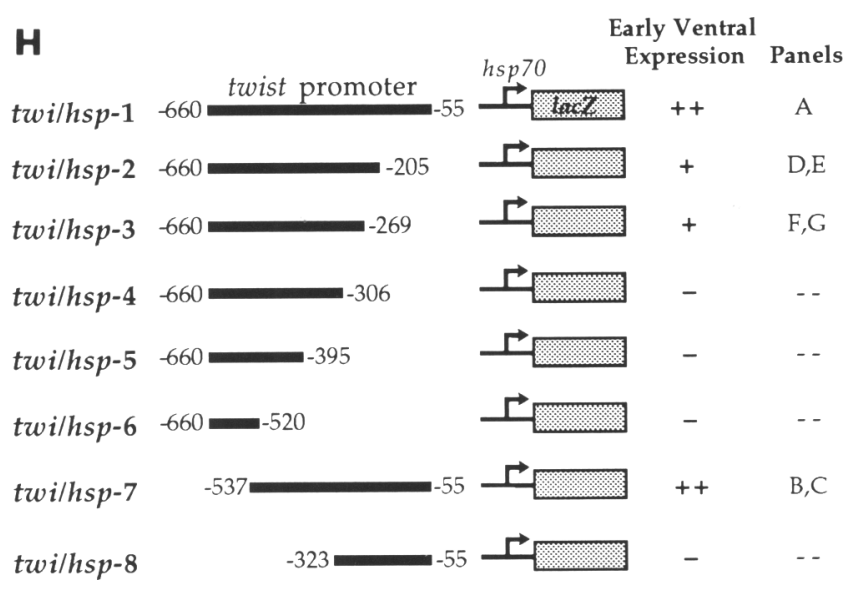

Figure 3. Ventral activation of a heterologous $h s p 70$ promoter. Expression patterns were revealed by in situ hybridization to whole-mount embryos. Embryos are oriented so that anterior is to the left; in the sagittal views, the dorsal side is up. $(A, B, D, F)$ Sagittal views; $(C, E, G)$ ventral views. The various constructs used are shown in $H$. $(A)$ twi/hsp $1 ;(B, C)$ twi/hsp-7; $(D, E)$ twi/ $h s p-2 ;(F, G) t w i / h s p-3$. Note that the level of expression in $D-G$ is much lower than that in $A-C$. Embryos were stained for longer times than those in Figs. 1 and 2. Further deletions (illustrated in $H$ ) abolish ventral expression (data not shown).

specific DNA-binding protein with a binding specificity very similar to that of the human transcription factor NF-кB. Presumably, therefore, $d l$ controls transcription by binding directly to cis-regulatory elements and influencing the rate of transcriptional initation. Thus, we expect to find one or more binding sites for the $d l$ protein in the VAR. Inspection of the 600-bp proximal promoter region reveals no perfect matches for the consensus sequence defined by Ip et al. (1991). However, we do find a single 9 of 10 match (nucleotides -299 to -290 ). This best match falls near the $3^{\prime}$ border of the VAR and is contained within one of the footprints in that region (Fig. 4C). Furthermore, this sequence falls within the 37-bp region that the $3^{\prime}$-deletion analysis in Figure 3 demonstrated to be necessary (but not sufficient) for ventral activation.

To test the idea that this site represents a binding site for the $d l$ transcription factor, a truncated $d l$ cDNA containing the sequences encoding the DNA-binding domain was expressed in Escherichia coli. The resulting protein was tested for binding to the twi promoter by a DNase I footprinting assay. As shown in Figure 6A, the bacterially expressed protein binds specifically to a 40 -bp region in the twi promoter, including the 9 of 10 match for the $d l$ consensus-binding site. This protection, which is the only such footprint observed in the VAR, extends in both directions to include additional weaker matches 


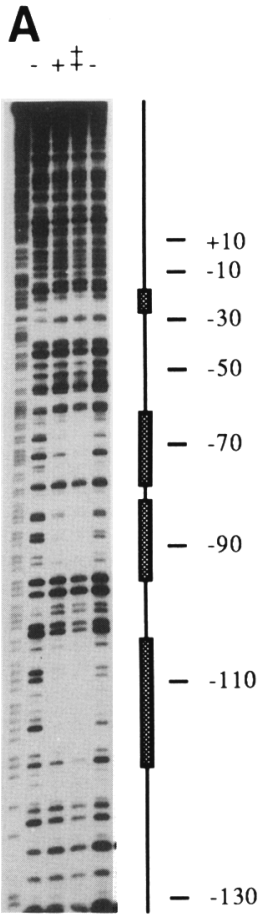

M1234

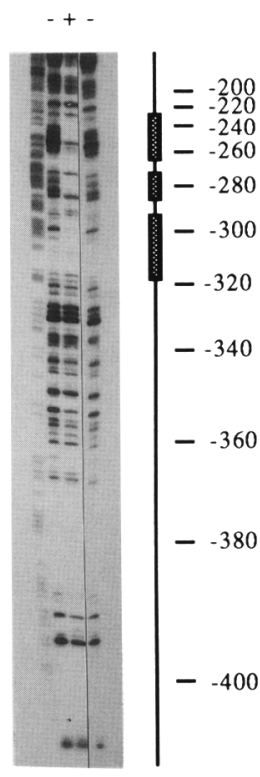

M567

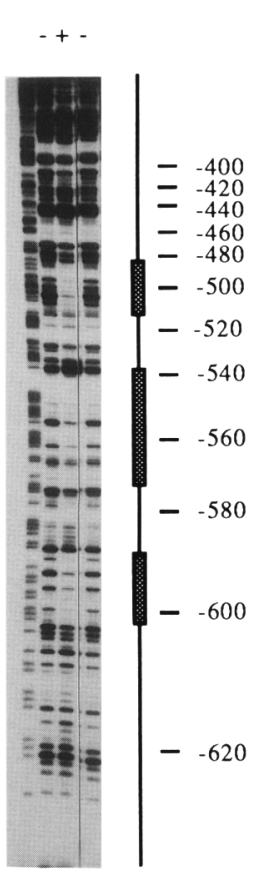

M8910

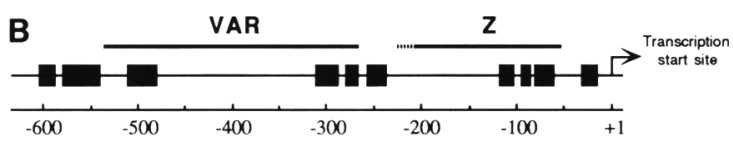

C

-679 caAaAagaat ataActtatt tccatatcat tTcaAgttTt taagtTaAat

-629 TCTTGTCTAA ACCTAAACTA ACGCTGCAAA CAACAacatT CAATGAagtT

-579 TACTAAATGT TCAATTGGGA ATTGCAAAAC AAAATCTTTT CTACCAGAAT

-529 GCAATTTTCA GGAAATGCTT TTATGTAATA AACATAATTT ATCATTACTC

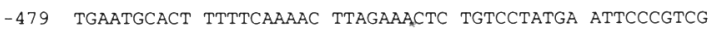

429 ATCCAAAGAT ATTCTCAATC CCCTTTTtTTG AatCAACAAG TAAAATATTT

-379 TAaAaATTGC CGACAATTCC CCTCGTATTC CCCGTCCCGC ATCCCAACAC

329 GCATACTTCC CAGGCATTTT CCCAAATCGa GagaAaACCC AaAGAataAC

279 CCAAGAGAAA CAGAAAAATC CAGAGGGTCG AGTCAAGGCT CTCTTCAATT

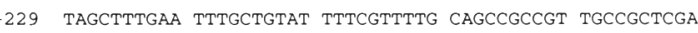

-179 GaAaAtcGaA atCCCCCAGC CTGACGTCAT ACCTGCCGAt GCCGCAGCtT 129 CCGCCATTGA GTGGGAGCGG GATGGCAAGA CAAGCGAGCG AGCGGaCGA

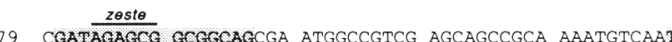
$29 \frac{z e s t \theta}{\text { TTGAGCAATE ECCGGAAGGA TCCTGCGTCA GTTGCGTTCC }}$

Figure 4. Footprint analysis of the 600-bp twi proximal promoter region. $(A)$ Three uniquely end-labeled footprinting probes were generated by using exonuclease III/mung bean nuclease deletion derivatives of the $t w i$ promoter region. These probes were 5 '-endlabeled at -155 (left), -434 (middle), or -648 (right). Probes were incubated with no extract (lanes $1,4,5,7,8,10$ ), $4 \mu \mathrm{l}$ of SK embryo extract (lanes 2,6,9), or $8 \mu$ l of SK embryo extract (lane 3). Reaction products were then displayed on a $6 \%$ sequencing gel. The approximate positions of the footprints discussed in the text are shown by the boxes at right. Lanes $M$ contain the appropriate $\mathrm{G}+\mathrm{A}$ chemical sequencing ladders. Additional data (not shown) indicates that there are no significant protections between -200 and -130 . $(B)$ The positions of the various protected regions are indicated by boxes. The lines above the sequence represent the two transcriptional elements inferred from our deletion analysis (in particular, Fig. 3). (C) Sequence of the twi proximal promoter region. The shading indicates the sequences protected from DNase I digestion by the crude Drosophila embryo extracts. The bracket under the sequence shows the extent of the footprint generated by bacterially expressed $d l-378$ protein (see Fig. 6). Also indicated are the $d l$ and zeste recognition sequences discussed in the text. The zeste consensus-binding site is (C/T)GAG(C/T)G (Biggin and Tjian 1989). The $d l$ consensus binding site is GGGA(A/G)A(A/T)(A/C)C(A/C) (Ip et al. 1991). The central $d l$ binding site is labeled in larger type because it represents the best match for the $d l$ consensus-binding sequence.

for the consensus sequence on either side of the best match (Fig. 6B). Thus, this protection seems to represent the binding of $d l$ protein to three consecutive sites within the VAR. Interestingly, all three sites contain one half-site that matches the zen consensus perfectly and a second half-site of lesser quality. In conclusion, the activity of the VAR appears to be the result, at least in part, of its ability to serve as a binding site for the $d l$ transcription factor. However, additional sequences (between -537 and -323 ) upstream of the $d l$-binding sites are also required for ventral activation.

\section{Discussion}

Our data show that a region of twi extending from -1829 to +236 can direct a pattern of transcription that is very similar to the wild-type twi pattern of transcrip- tion. Furthermore, these experiments allow us to identify a small region of the $t w i$ promoter that can mediate the $d l$-dependent ventral activation of a heterologous promoter. This region, which we have termed the VAR, lies between -537 and -269 relative to the twi transcriptional start site. The VAR includes a 40-bp region (from -277 to -317 ) that is specifically protected from DNase I digestion by bacterially expressed $d l$ protein. A 37 -bp deletion (from -269 to -306 ) that removes most of this region inactivates the VAR. Thus, the $d l$-binding region is essential for ventral-specific transcriptional activation.

DNase I footprinting experiments with Drosophila embryo extracts reveal that the VAR overlaps two clusters of protected regions centered at about -275 and -540 . The proteins responsible for these footprints remain largely uncharacterized. However, the most $5^{\prime}$ footprint in the cluster centered around -275 is con- 
Figure 5. In vitro transcription of the $t w i$ promoter. $(A)$ Reactions $(25 \mu \mathrm{l})$ were programmed with $150 \mathrm{ng}$ of one of the twi promoter deletion mutants shown at the bottom. In addition, reactions contained 6 $\mu l$ of HP transcription extract. The arrow indicates the position of the correctly initiated twi transcripts. $(B)$ Reactions $(25 \mu \mathrm{l})$ were programmed with $50 \mathrm{ng}$ of the indicated twi promoter 5 '-deletion mutants and $100 \mathrm{ng}$ (lanes $1-9$ ) or $400 \mathrm{ng}$ (lanes 10 12) of a construct containing a $-555^{\prime}$ deletion of the $A d h$ proximal promoter. In addition, the reactions contained $2 \mu \mathrm{l}$ of SK extract (lanes 1-6) or $6 \mu$ l of HP extract (lanes 7-12), as well as the indicated amounts of histone $\mathrm{H} 1$. The positions of the correctly initiated twi and Adh transcripts are indicated by arrows. For all experiments in $A$ and $B$, transcription products were visualized by S1 nuclease analysis as described in Materials and methods.
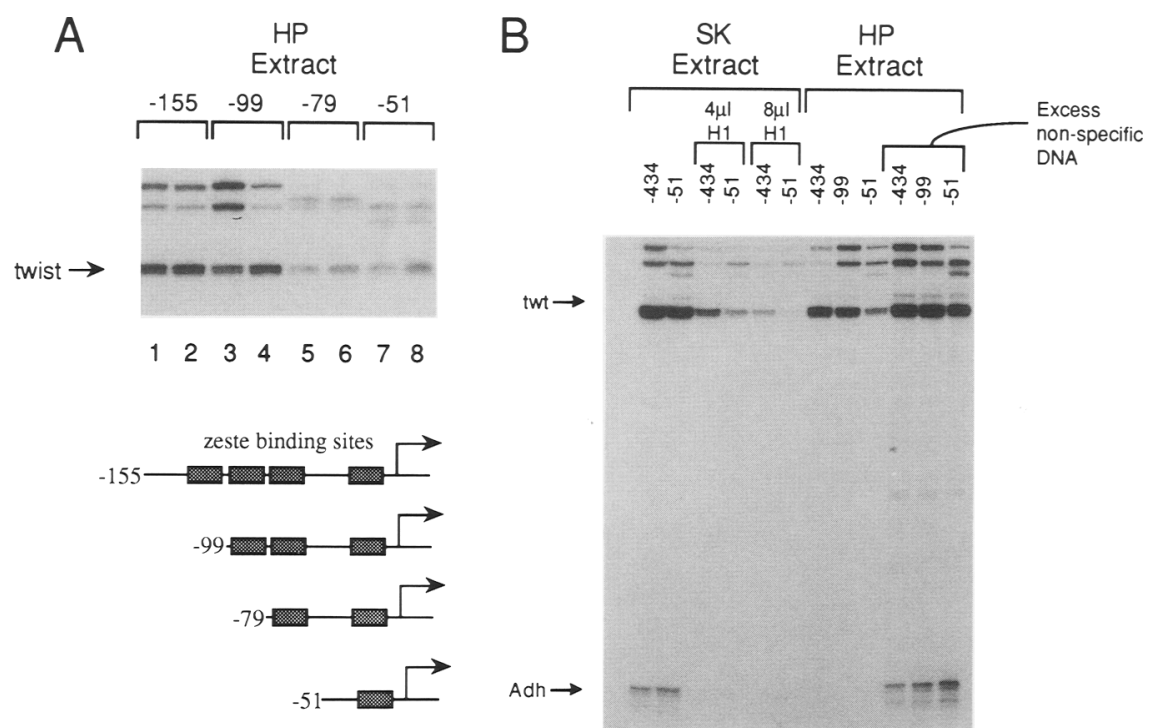

tained within the region that is protected by bacterially expressed $d l$ protein. We postulate that this footprint is the result of $d l$ gene product in the embryo nuclear extracts. Thus, this experiment may represent the first detection of the active embryonic $d l$ protein. We believe that the footprint obtained with the bacterial protein represents protection over three closely spaced $d I$-binding sites (Figs. 4C and 6). However, the embryo extracts seem to contain another protein that binds to a site overlapping the most $3^{\prime} d l$ recognition sequence (Fig. 4A-C).
In the presence of this protein, $d l$ seems to be excluded from this third site.

Although our data strongly support the idea that the $d l$-binding sites are required for ventral activation, they also provide support for the notion that sequences in addition to the $d l$-binding sites are required to create a ventral activator. For example, the construct containing nucleotides -323 to -55 linked to the $h s p 70$ basal level promoter (twi/hsp-8; Fig. 3 ) is not active even though it contains the entire $d l$-binding region. In contrast, twi/
Figure 6. $d l$ protein binds to the ventral activator region. $(A)$ A probe that had been 5 '-end-labeled at -434 was incubated with a bacterial extract containing the $d l-378$ protein or a control extract of nonexpressing bacteria. The probes were then digested with DNase I before electrophoresis on a $6 \%$ sequencing gel. (Lane 1) no protein; (lanes 2,3) $25 \mu \mathrm{l}$ of the control extract; (lane 4) $10 \mu$ l of the control extract; (lanes 5,6$) 25 \mu \mathrm{l}$ of the $d l-378$ extract; (lane 7) $10 \mu \mathrm{l}$ of $d l-378$ extract; (lane 8 ) no protein. Lanes 2 and 3 and 5 and 6 differ in the extent of DNase I digestion, with lanes 3 and 6 being underdigested relative to lanes 2 and 5 . Lane $M$ contains the $\mathrm{A}+\mathrm{G}$ chemical sequencing ladder. $(B)$ Alignment of the $d l$ recognition sequences in the $t w i$ VAR, with the consensus-binding site derived from the sites in the zen ventral repressor (Ip et al. 1991). Stars indicate matches with the consensus.

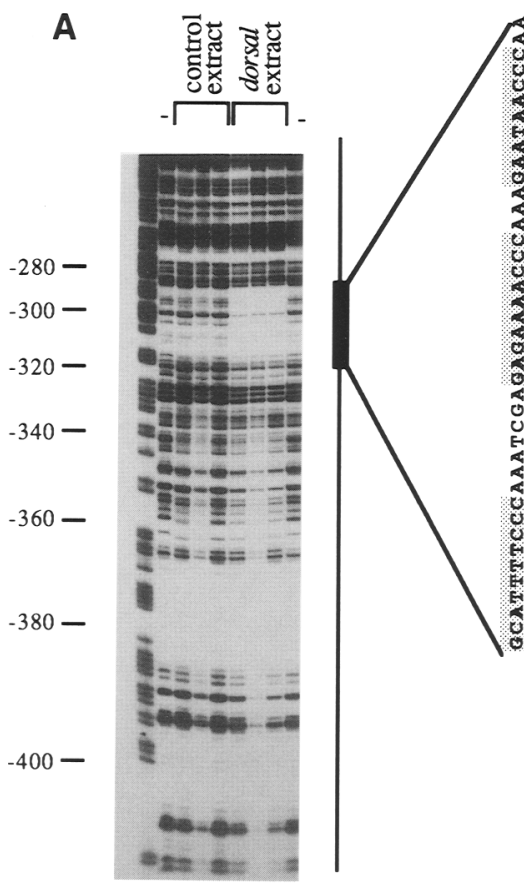

M12345678

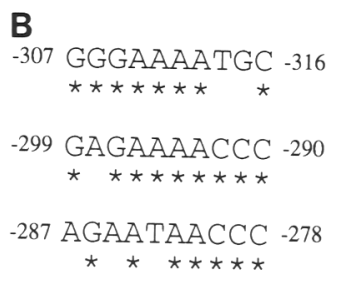

GGGAA ${ }_{\mathrm{G}}^{\mathrm{A}} \stackrel{\mathrm{T}}{\mathrm{T}} \mathrm{C} \stackrel{\mathrm{A}}{\mathrm{C}}$ zen consensus 
hsp-7, which contains additional sequences upstream of the essential $d l$-binding sites, displays ventral activity. These additional sequences do not bind $d l$ with any detectable affinity. This finding may reflect the ability of $d l$ to serve as both a transcriptional activator (e.g., for twi and sna) and a transcriptional repressor (e.g., for zen and $d p p)$. It is likely that sequence elements outside the $d l$ recognition sequence are responsible for modifying the activity of bound $d l$ protein and converting it into either an activator or a repressor. One intriguing possibility is that the DNase I-protected region at the $5^{\prime}$ end of the VAR serves the function of turning $d l$ protein bound to the $3^{\prime}$ end of the VAR into an activator, and we are currently testing this possibility.

The differential activity of the zen ventral repressor and the twi ventral activator could also be due to the existence of multiple dl-containing heteromeric complexes in the Drosophila embryo. As mentioned above, the $d l$ protein bears sequence similarity to the human transcription factor NF-kB, and the two proteins have similar binding site specificities. The predominant DNA-binding form of NF- $\mathrm{kB}$ may be a heterodimer between two polypeptides termed p50 and p65 (Baeuerle and Baltimore 1989; Nolan et al. 1991). In all of the experiments with bacterially expressed $d l$, the observed DNA-binding affinity is extremely low (Ip et al. 1991; J. Shirokawa and A.J. Courey, unpubl.). Although this could be the result of partially denatured bacterially expressed protein, it may also suggest that $d l$ protein normally binds to DNA and regulates transcription as a part of heteromeric complexes with other polypeptides. To test these possibilities, it will be necessary to use biochemical methods to examine the protein-protein interactions between the $d l$ protein and other proteins present in extracts of Drosophila embryos. These studies will be facilitated greatly by our ability to detect $d l$-binding activity in crude Drosophila embryo extracts.

Wild-type levels of $t w i$ expression appear to require sequences upstream and downstream of the VAR. In particular, sequences between -205 and -55 (element $z$ ) were found to boost the activity of the VAR severalfold. Sequences between -1829 and -920 also stimulate the activity of the twi promoter. In addition, these distal sequences are required to broaden the ventral domain, for early terminal expression, and to repress aberrant head expression of $t w i$. We are currently carrying out experiments to determine whether this region can serve as an independent ventral activator region.

Element $z$ contains multiple tandem binding sites for the zeste transcription factor. Our in vitro transcription experiments show that under certain conditions, promoter activity decreases significantly with the deletion of two of the zeste-binding sites. Specifically, we found that the activity of the region containing the zeste-binding sites was critically dependent on the presence of histone $\mathrm{Hl}$ in the transcription system. Thus, as with the Drosophila GAGA factor (Croston et al. 1991; Kerrigan et al. 1991), zeste protein may stimulate transcription by excluding $\mathrm{H} 1$ from the promoter region.

zeste is required for the process of transvection, whereby an enhancer element on one chromosome activates a promoter element on the homologous chromosome (Wu and Goldberg 1989). Transvection requires normal chromosome pairing, and the zeste protein may act as a link to hold the promoter and remote enhancer together (Benson and Pirrotta 1988; Biggin et al. 1988). Thus, the zeste-binding sites in element $\mathrm{z}$ may mediate the effects of the more distal regulatory elements in the twi promoter by anchoring a chromosomal loop. In this regard, it is interesting to note the presence of an additional possible zeste-binding site just $3^{\prime}$ of the $d l$-binding sites in the VAR (Fig. 4C).

In conclusion, the experiments described in this paper should lead to an understanding of the biochemical basis of transcriptional activation and repression. In addition, they will illuminate the mechanisms used to generate discrete germ layers during development. Ultimately, we hope to learn how the positional information present in morphogenic gradients can result in the formation of complex multicellular organisms.

\section{Materials and methods}

P-element-mediated transformation and analysis of expression patterns

Germ-line transformation was carried out as described previously (Rubin and Spradling 1982). Constructs were injected into $w^{1118}$ flies with $\mathrm{p} \pi 25.7 \mathrm{wc}$ helper DNA (Karess and Rubin 1984). Multiple independent lines were established for each P-element construct and examined for $\beta$-galactosidase activity. In situ hybridization to whole-mount embryos was carried out according to standard procedures (Tautz and Pfeifle 1989) with digoxygenin-ATP-labeled probes (Genius kit, Boehringer Mannheim). The stained embryos were examined and photographed with a Zeiss Axiophot microscope and Nomarski optics. Embryo stages are according to Campos-Ortega and Hartenstein (1985).

To examine the $d l$ dependence of the various P-element constructs we used a strain containing a balanced null allele of $d l$ $\left(d l^{1}\right.$, cn sca/Cyo, DTS-100). $w^{111}$ male flies homozygous for a $\mathrm{P}$ insertion were crossed to females homozygous for $d l^{1}$. The resulting embryos were analyzed as described above.

\section{Plasmid constructions}

We have constructed two series of plasmids for use in P-element-mediated germ-line transformation. The first contains various lengths of $t$ wi $5^{\prime}$-flanking region fused to the $E$. coli lacZ gene. These plasmids were constructed in two steps. First, a BamHI-PstI fragment (from -11 to +236 ) was cloned into the BamHI site of cosPwhite $\beta$-gal (Pirotta 1988) by adding a BgIII linker (CAGATCTG, New England Biolabs ) to the PstI end. This results in an in-frame fusion of the first 26 amino acids of $t w i$ to the eighth amino acid of $\beta$-galactosidase, with the additional sequence CCA GAT CCC (Pro Asp Pro) at the junction. This construct is called $-11 \mathrm{twi} / \mathrm{lac} Z$. In the next step, BamHIBamHI, PstI-BamHI, or XmnI-BamHI fragments were cloned just upstream of the $B a m H I$ site in -11 twi/lacZ to generate $-5000 \mathrm{twi} / \mathrm{lacZ},-1829 \mathrm{twi} / \mathrm{lacZ}$, and $-920 \mathrm{twi} / \mathrm{lacZ}$, respectively. The $-648 \mathrm{twi} / \mathrm{lac} Z$ and $-269 \mathrm{twi} / \mathrm{lac} Z$ constructs were made similarly, using some of the exonuclease III and mung bean nuclease 5 ' deletions of the twi promoter that were generated for the in vitro transcription and footprinting analysis (see below). 
The second series of P-element plasmids contains deletions of the 660-bp proximal promoter region fused to an $h s p 70 / 1 a c Z$ gene in a P-element vector that was constructed by G. Liaw and J. Lengyel (unpubl.). This vector contains the $h s p 70$ promoter from -50 to the cap site fused to $1 a c Z$ (Hiromi and Gehring $1987)$ and a mini-white gene as a marker. The deletions of the 660-bp proximal promoter region shown in Figure $3 \mathrm{H}$ were generated by polymerase chain reaction (PCR). For each pair of primers, one has an overhang bearing an $\mathrm{XbaI}$ site and the other has a NotI overhang. This allows the PCR products to be cloned directionally into $\mathrm{XbaI}$ and NotI sites in the polylinker region of the vector.

The $-648,-434,-269,-155,-99,-79$, and $-515^{\prime}$-deletion mutations of the twi promoter used in the footprinting and in vitro transcription analysis and in the subsequent construction of some of the P-element constructs were generated by deletion with exonuclease III and mung bean nuclease. A PstI fragment extending from -1829 to +236 of the $t w i$ gene was subjected to unidirectional exonucleolytic digestion and the various size products were cloned in the Bluescript $\mathrm{SK}+$ vector between the EcoRV and PstI sites.

Drosophila embryo extracts, in vitro transcription, and DNase I footprinting

SK and HP extracts were prepared from 0- to 12 -hr-old Oregon $\mathbf{R}$ embryos as described previously (Heiermann and Pongs 1985; Soeller et al. 1988; Kerrigan et al. 1991). Drosophila embryo histone $\mathrm{Hl}$ was a gift of J. Kadonaga. In vitro transcription reactions and S1 nuclease analysis were carried out as described previously (Biggin and Tiian 1988). The single-stranded S1 probe used for analysis of the $t w i$ transcripts was generated by cleaving the $-995^{\prime}$ deletion of the twi promoter with HindIII and $X b a I$, labeling with polynucleotide kinase, and isolating the nonsense strand on a strand-separating gel. The correctly initiated transcript is expected to give a 263 -bp protected species. The -55 deletion of the Adh proximal promoter and the S1 probe used to analyze its transcripts are as described in Biggin and Tjian (1988). DNase I footprint reactions were carried out as described previously (Heberlein et al. 1985). Footprinting reactions with embryo extracts contained $1 \mu \mathrm{l}$ of poly[d(I-C)] (10 $\mathrm{OD}_{260} \mathrm{U} / \mathrm{ml}$ ) as nonspecific competitor, whereas footprinting reactions with bacterial proteins contained no competitor.

\section{Expression of $\mathrm{dl}$ protein in $\mathrm{E}$. coli}

The truncated $d l$ protein ( $d l-378)$ was expressed by using a $T 7$ expression construct that is essentially identical to the one described by Ip et al. (1991). Expression was carried out with a strain of $E$. coli (BL21) lysogenic for a $\lambda$ phage containing the gene for T7 RNA polymerase under the control of the lac promoter. Cells were grown to mid- $\log \left(O D_{550}=0.5\right)$, and expression was induced with $0.4 \mathrm{~mm}$ IPTG. After an additional hour at $37^{\circ} \mathrm{C}$, inclusion body extracts were prepared as described previously (Bohmann and Tiian 1989).

\section{Acknowledgments}

We thank Ruth Steward for providing us with the $d l$ cDNA, Mark Biggin for providing purified zeste protein, and Jim Kadonaga for supplying Drosophila histone H1. We also thank Judy Lengyel and Gwo-Jen Liaw for providing us with their hsp 70 promoter P-element vector before publication. In addition, we gratefully acknowledge Steve Crews and John Nambu for providing their advice and expertise, particularly in the techniques of germ-line transformation and in situ hybridization. Finally, we thank Steve Smale, John Nambu, Mike Carey, and
Sabeeha Merchant for their careful and critical reading of this manuscript. This work was supported by grant GM44522 from the National Institutes of Health.

The publication costs of this article were defrayed in part by payment of page charges. This article must therefore be hereby marked "advertisement" in accordance with 18 USC section 1734 solely to indicate this fact.

\section{Note added in proof}

Since the submission of this paper, Thisse et al. (Cell 65: 11911201) have published an analysis of the $t w i$ promoter that reaches some of the same conclusions reported here. These investigators present evidence suggesting that $d l$ protein binds specifically to the twi promoter, and in tissue culture cell cotransfection assays, they show that $d l$ can activate $t w i$ transcription. The findings of Jiang et al. (this issue) overlap and complement the studies reported in this paper. In addition to the proximal VAR that is the focus of our study, these researchers have also identified a more distal $\mathrm{dl}$-binding element (from 1.2 to $0.8 \mathrm{~kb}$ upstream of the twi transcriptional start site) that can independently activate ventral specific transcription from the twi promoter.

\section{References}

Baeuerle, P.A. and D. Baltimore. 1989. A $65 \mathrm{kD}$ subunit of active

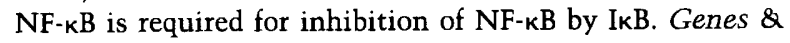
Dev. 3: 1689-1698.

Benson, M. and V. Pirrotta. 1988. The Drosophila zeste protein binds cooperatively to sites in many gene regulatory regions: Implications for transvection and gene regulation. EMBO J. 7: 3907-3915.

Biggin, M.D. and R. Tjian. 1988. Transcription factors that activate the Ultrabithorax promoter in developmentally staged extracts. Cell 53: 699-711.

1989. Transcription factors and the control of Drosophila development. Trends Genet. 5: 377-383.

Biggin, M.D., S. Bickel, M. Benson, V. Pirrotta, and R. Tjian. 1988. zeste encodes a sequence-specific transcription factor that activates the Ultrabithorax promoter in vitro. Cell 53: 713-722.

Bohmann, D. and R. Tiian. 1989. Biochemical analysis of transcriptional activation by jun: Differential activity of $c-$ and v-jun. Cell 59: 709-717.

Campos-Ortega, J.A. and V. Hartenstein. 1985. The embryonic development of Drosophila melanogaster. Springer-Verlag, Berlin.

Croston, G.E., L.A. Kerrigan, L.M. Lira, D.R. Marshak, and J.T. Kadonaga. 1991. Sequence-specific antirepression of histone H1-mediated inhibition of basal RNA polymerase II transcription. Science 251: 643-649.

Ghosh, S., A.M. Gifford, L.R. Riviere, P. Tempst, G.P. Nolan, and D. Baltimore. 1990. Cloning of the p50 DNA binding subunit of NF-kB: Homology to rel and dorsal. Cell 62: 1019-1029.

Govind, S. and R. Steward. 1991. Dorsoventral pattern formation in Drosophila: Signal transduction and nuclear targeting. Trends Genet. 7: 119-125.

Heberlein, U., B. England, and R. Tjian. 1985. Characterization of Drosophila transcription factors that activate the tandem promoters of the alcohol dehydrogenase gene. Cell 41: 965977.

Heiermann, R. and O. Pongs. 1985. In vitro transcription with extracts of nuclei of Drosophila embryo. Nucleic Acids Res. 13: 2709-2730. 
Hiromi, Y. and W.J. Gehring. 1987. Regulation and function of the Drosophila segmentation gene fushi tarazu. Cell 50: $963-974$.

Ingham, P.W. 1988. The molecular genetics of embryonic pattern formation in Drosophila. Nature 335: 25-34.

Ip, Y.T., R. Kraut, M. Levine, and C.A. Rushlow. 1991. The dorsal morphogen is a sequence-specific DNA-binding protein that interacts with a long-range repression element in Drosophila. Cell 64: 439-446.

Karess, R.E. and G. Rubin. 1984. Analysis of P transposable element functions in Drosophila. Cell 38: 135-146.

Kerrigan, L.A., L.M. Croston, J.T. Lira, and J.T. Kadonaga. 1991. Sequence-specific transcriptional antirepression of the Drosophila Krüppel gene by the GAGA factor. I. Biol. Chem. 266: 574-582.

Kieran, M., V. Blank, F. Logeat, J. Vandekerckhove, F. Lotspeich, O. Le Bail, M.B. Urban, P. Kourilsky, P.A. Baeuerle, and A. Israel. 1990. The DNA binding subunit of NF- $\mathrm{kB}$ is identical to factor KBF1 and homologous to the rel oncogene product. Cell 62: 1007-1018.

Murre, C., P.S. McCaw, and D. Baltimore. 1989. A new DNA binding and dimerization motif in immunoglobulin enhancer binding, daughterless, MyoD, and myc proteins. Cell 56: 777-783.

Nolan, G.P., S. Ghosh, S. Liou, P. Tempst, and D. Baltimore. 1991. DNA binding and IKB inhibition of the cloned p65 subunit of NF-кB, a rel-related polypeptide. Cell 64: 961969.

Pirotta, V. 1988. Vectors for P-mediated transformation in Drosophila. In Vectors, a survey of molecular cloning vectors and their uses (ed. R.L. Rodriguez and D.T. Denhardt), pp. 437-456. Butterworths, Boston.

Roth, S., D. Stein, and C. Nüsslein-Volhard. 1989. A gradient of nuclear localization of the dorsal protein determines dorsoventral pattern in the Drosophila embryo. Cell 59: 11891202.

Rubin, G.M. and A.C. Spradling. 1982. Genetic transformation of Drosophila with transposable element vectors. Science 218: 348-353.

Rushlow, C.A., K. Han, J.L. Manley, and M. Levine. 1989. The graded distribution of the dorsal morphogen is initiated by selective nuclear transport in Drosophila. Cell 59: 11651177.

Soeller, W., S.J. Poole, and T. Kornberg. 1988. In vitro transcription of the Drosophila engrailed gene. Genes \& Dev. 2: 6881 .

Steward, R. 1987. Dorsal, an embryonic polarity gene in Drosophila is homologous to the vertebrate proto-oncogene, c-rel. Science 238: 692-694.

-1989. Relocalization of the dorsal protein from the cytoplasm to the nucleus correlates with its function. Cell 59: 1179-1188.

Tautz, D. and C. Pfeifle. 1989. A non-radioactive in situ hybridization method for the localization of specific RNAs in Drosophila embryos reveals translation control of the segmentation gene hunchback. Chromosoma 98: 81-85.

Thisse, B., C. Stoetzel, M. El Messal, and F. Perrin-Schmitt. 1987. Genes of the Drosophila maternal dorsal group control the specific expression of the zygotic gene twist in presumptive mesodermal cells. Genes \& Dev. 1: 709-715.

Thisse, B., C. Stoetzel, C. Gorostiza-Thisse, and F. PerrinSchmitt. 1988. Sequence of the twist gene and nuclear localization of its protein in endomesodermal cells of early Drosophila embryos. EMBO I. 7: 2175-2183.

Wu, C. and M.L. Goldberg. 1989. The Drosophila zeste gene and transvection. Trends Genet. 5: 189-194. 


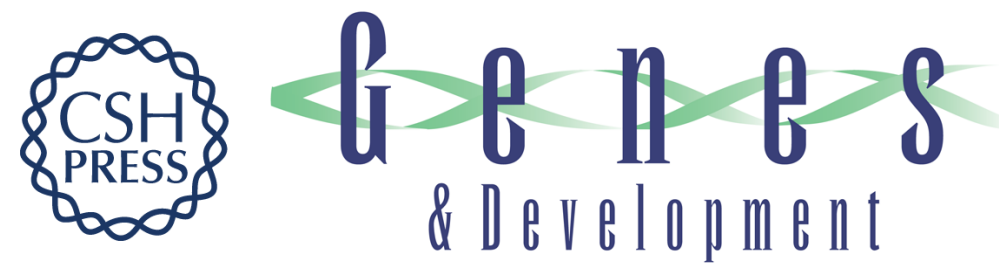

\section{Functional analysis of the Drosophila twist promoter reveals a dorsal-binding ventral activator region.}

D J Pan, J D Huang and A J Courey

Genes Dev. 1991, 5:

Access the most recent version at doi:10.1101/gad.5.10.1892

References This article cites 29 articles, 7 of which can be accessed free at:

http://genesdev.cshlp.org/content/5/10/1892.full.html\#ref-list-1

License

Email Alerting

Service

Receive free email alerts when new articles cite this article - sign up in the box at the top right corner of the article or click here.

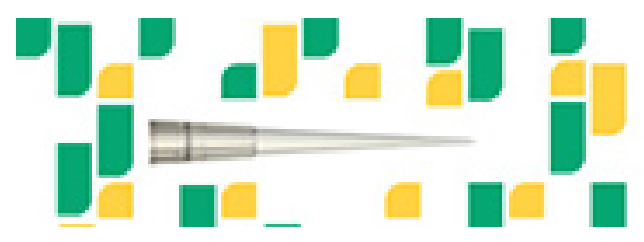

Focused on your science.

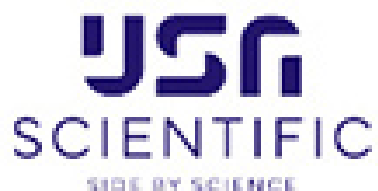

Copyright (c) Cold Spring Harbor Laboratory Press 